\title{
Phase Conjugated Twin Waves Based Transmission in Few Modes Fibers
}

\author{
J. S. Tavares ${ }^{1}$, L. M. Pessoa ${ }^{1}$, H. M. Salgado ${ }^{1,2}$ \\ ${ }^{1}$ INESC TEC \\ ${ }^{2}$ Faculty of Engineering, University of Porto, \\ Porto, Portugal \\ Tel: (+351) 222094 000, Fax: (+351) 222094050 \\ e-mail:\{joana.s.tavares, luis.m.pessoa\}@inescporto.pt, \{hsalgado\}@fe.up.pt
}

\begin{abstract}
Spatial division multiplexing (SDM) is seen as an effective technique to overcome the present limits of fiber capacity and satisfy future bandwidth requirements, by substantially increasing optical transport capacities [1-5]. Similarly to single-mode fiber based modern telecommunication systems, the nonlinear penalties will eventually become the ultimate limiting factor in SDM systems, arising from crosstalk effects in multimode core or few mode fibers, which generate intramodal and intermodal nonlinearities. Here we explore the concept of conjugated twin waves in few mode fibers based SDM optical systems, as a means to overcome these nonlinear impairments and assess the performance of SDM systems for different PCTW configurations and coupling regimes. Our numerical simulation results show that this technique is an effective means to overcome the nonlinear impairments arising in mode division multiplexed systems, improving performance by up to $10 \mathrm{~dB}$, in the strong coupling regime.
\end{abstract}

Keywords: fiber nonlinearity, multimode fiber, phase conjugated twin waves, space-division multiplexing.

\section{INTRODUCTION}

In this work we investigate the concept of phase conjugated twin waves (PCTWs) in spatial division multiplexing (SDM) optical systems, which has recently been proved to cancel out signal-to-signal nonlinear interactions in single-mode fibers by coherently superimposing of a pair of phase-conjugated twin waves, as a means to overcome the nonlinear impairments arising in mode division multiplexed systems, in a practical and sustainable way, paving the way for optical transmission capacities beyond next-generation systems [6]. Specifically, we investigate the application of the PCTW technique on different SDM scenarios, namely PDMwise PCTW, by propagating each pair of PCTWs on different spatial modes; a single polarization (SP) generalization of PCTW that consists of using an orthogonal dimension (space) to transmit the twin wave; and a dual polarization (DP) generalization of PCTW, assuming that the signal and its conjugated twin are polarization division multiplexed (PDM) signals propagating on orthogonal dimensions.

First, we describe the principle of PCTWs in different scenarios. Then, we present an overview of the numerical simulation framework comprising simulation parameters, and finally, the obtained numerical simulation results are presented and discussed.

\section{PCTW PRINCIPLES}

Single mode (SM)-PCTW-based transmission consists of co-propagating an optical signal and its complex conjugate on the orthogonal polarization of the same optical carrier. The nonlinear distortions experienced by the pair of PCTWs are anti-correlated, so that the signal-to-signal interactions can be cancelled by coherently

Table 1. Overview of different SDM-PCTW scenarios.

\begin{tabular}{|c|c|c|c|}
\hline & $\begin{array}{l}\text { PDM-wise } \\
\text { PCTW }\end{array}$ & $\begin{array}{l}\text { Mode-wise } \\
\text { SP-PCTW }\end{array}$ & $\begin{array}{l}\text { Mode-wise } \\
\text { DP-PCTW }\end{array}$ \\
\hline LP01 & 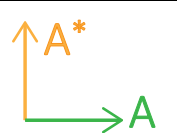 & $\longrightarrow A$ & $\stackrel{\wedge}{\longrightarrow} \stackrel{A}{\longrightarrow}$ \\
\hline LP11a & $\stackrel{\mathrm{B}^{*}}{\longrightarrow} \mathrm{B}$ & $\longrightarrow A^{*}$ & 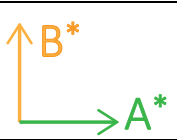 \\
\hline LP02 & $\stackrel{\mathrm{C}^{*}}{\longrightarrow} \mathrm{C}$ & $\longrightarrow$ B & $\stackrel{\uparrow}{\longrightarrow} C$ \\
\hline LP21a & 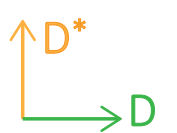 & $\longrightarrow \mathrm{B}^{*}$ & $\stackrel{D^{*}}{\longrightarrow} C^{*}$ \\
\hline
\end{tabular}


superimposing the received signals, although incurring a spectral efficiency loss of one half [6]. This technique also requires a symmetric dispersion map, which can be obtained by performing electronic dispersion precompensation. In this configuration the transmitted pair of PCTWs consists of $A_{x}=A$ and $A_{y}=A_{x}{ }^{*}$. At the receiver side the original signal is recovered by employing $A_{x}+A_{y}{ }^{*}=2 A$, hence the halved spectral efficiency.

We next present a description of the three investigated multimode PCTW scenarios, which are summarized on Table 1, considering 4 spatial modes.

\subsection{SDM-PCTW scenarios}

We propose a configuration, which we call PDM-wise PCTW, whereby each pair of PCTWs is propagated on a single spatial mode, different pairs being sent on $m$ distinct spatial modes. This is a natural extension of the previous single-mode PCTW.

A different approach has been proposed in [7]. Instead of using an orthogonal polarization to transmit the complex conjugate of a signal as in the original PCTW concept, in this case the twin wave is propagated on a different orthogonal dimension (i.e. space). It has been argued that this technique can be particularly beneficial to ensure secure communications [6]. This is described here as mode-wise SP-PCTW.

Additionally, Liu et al. also proposed the extension of the previous case to vector waves [7]. In this scenario the signal and its conjugated twin are PDM signals (consisting of two independent polarization components), propagating on orthogonal dimensions (i.e. space).. This is described here as mode-wise DP-PCTW. However, no performance results have been given regarding the last two proposed scenarios.

\section{NUMERICAL SIMULATIONS}

Our numerical simulations considered a 32-Gbaud PCTW-QPSK signal, consisting of $2^{13}$ modulated symbols. Each PCTW-QPSK signal was filtered by a squared root raised cosine (SRRC) filter with a 0.1 roll-off factor before pre-compensation for half of the fiber link dispersion.

The simulations were based on the standard symmetric split-step Fourier method to solve the generalized Manakov equations for multimode fibers [8], using a simulation step of $1000 \mathrm{~m}$. The transmission line consists of $10100-\mathrm{km}$ fiber spans, each span being followed by an erbium-doped fiber amplifier (EDFA) that compensates for the span losses. ASE (amplified spontaneous emission) noise was also added by each EDFA, with a noise figure of $5 \mathrm{~dB}$. We considered a step-index few-mode fiber (FMF), with a core radius of $6 \mu \mathrm{m}$ and a fiber loss of $0.2 \mathrm{~dB} / \mathrm{km}$. The nonlinear coefficient, numerical aperture, and V parameter at $1550 \mathrm{~nm}$ used were $1.4 \mathrm{~W}^{-1} \mathrm{~km}^{-1}, 0.2$, and 5 , respectively. With this configuration 6 spatial modes are supported by the fiber (LP01, LP11a, LP11b, LP02, LP21a, and LP21b). Dispersion and differential mode group delay (DMGD) calculated for each mode are presented on Table 2.

At the receiver side, the received signals are post-dispersion compensated for the rest of the transmission link, followed by SRRC filtering. After phase estimation, the received signals are coherently super-positioned.

Table 2. Fiber dispersion and differential mode group delay for different spatial modes.

\begin{tabular}{|c|c|c|}
\hline Mode & $\mathrm{D}(\mathrm{ps} / \mathrm{nm} / \mathrm{km})$ & DMGD $(\mathrm{ns} / \mathrm{km})$ \\
\hline LP01 & 25.1 & 0 \\
\hline LP11 & 27.4 & 6.5 \\
\hline LP02 & -2.5 & 9.9 \\
\hline LP21 & 20.9 & 12.4 \\
\hline
\end{tabular}

\section{RESULTS}

In this section we present the results of our numerical simulations considering three different scenarios. Furthermore, weak coupling and strong coupling regimes are also evaluated for each case, using the corresponding Manakov equations as proposed in [8].

In all our numerical simulations, multimode PCTW transmission consists of co-propagating 4 spatial modes (i.e. LP01, LP11a, LP02, and LP21a). System performance is evaluated by calculating the $\mathrm{Q}^{2}$-factor of the received signals (averaged for all the spatial modes) for different launch powers, after $1000 \mathrm{~km} \mathrm{fiber}$ transmission.

The attained results are presented in Figures 1 to 6 , corresponding to the scenarios summarized in Table 1, considering the weak and strong coupling regime. For each case we present the $\mathrm{Q}^{2}$-factor calculated before and after coherent superposition, i.e., for the SDM (circles) and PCTW signals (squares), respectively. Dashed lines represent AWGN (Additive White Gaussian Noise) limit for each curve. The right hand axis shows the $\mathrm{Q}^{2}$-factor improvement (triangles). 
Results show that in the strong coupling regime a performance improvement of $\sim 10 \mathrm{~dB}$ is achieved at $8 \mathrm{dBm}$ of launched power, for all three scenarios. In the weak coupling regime the highest $\mathrm{Q}^{2}$-improvement is $\sim 7 \mathrm{~dB}$ at $6 \mathrm{dBm}$ in the first scenario and less than $3 \mathrm{~dB}$ in the other two.

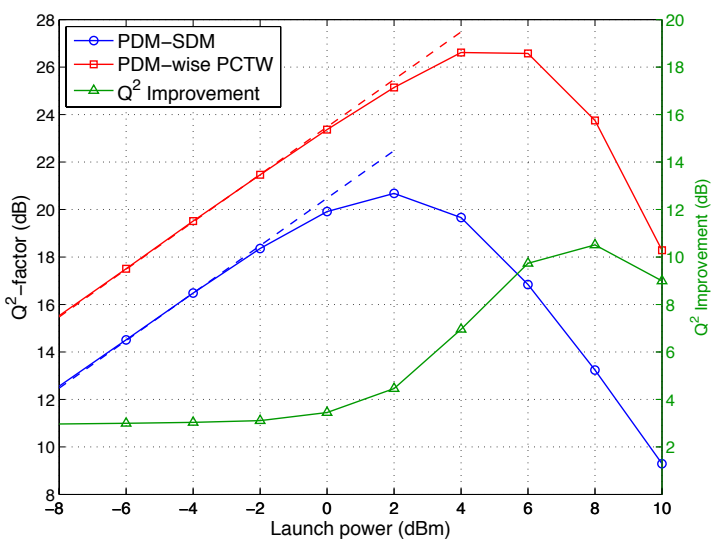

Figure 1. PDM-wise PCTW in strong coupling.

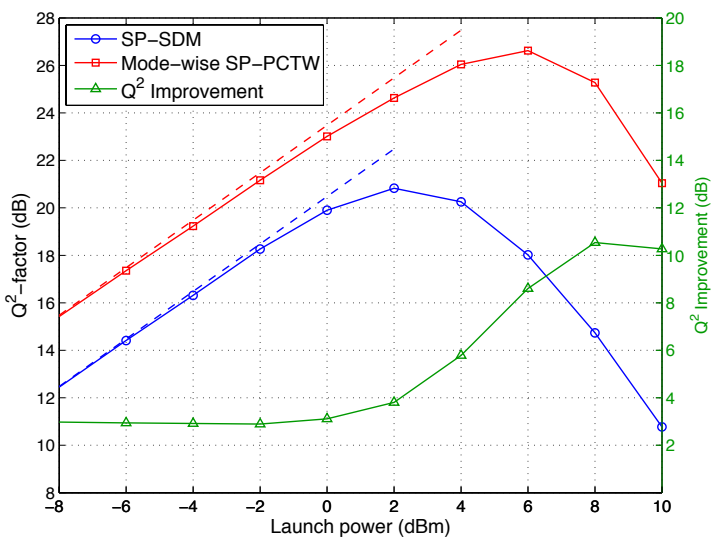

Figure 3. Mode-wise SP-PCTW in strong coupling.

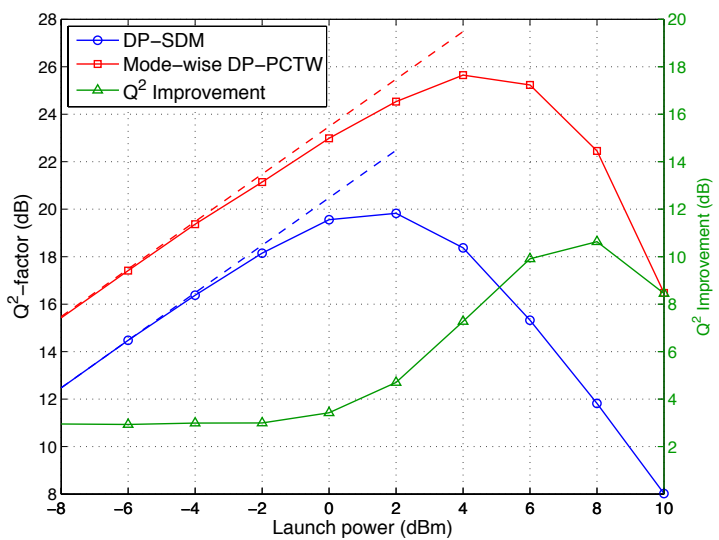

Figure 5. Mode-wise DP-PCTW in strong coupling.

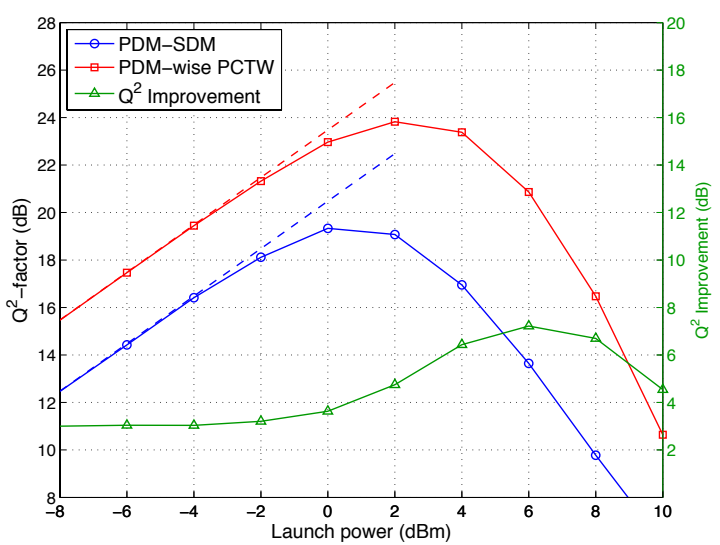

Figure 2. PDM-wise PCTW in weak coupling.

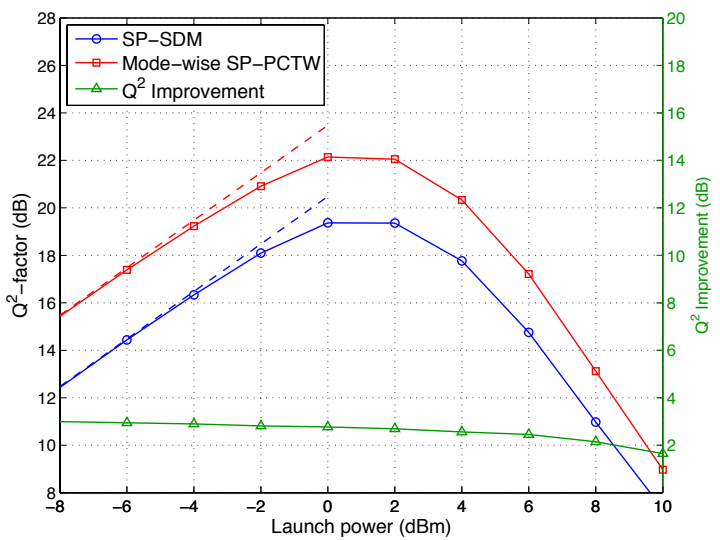

Figure 4. Mode-wise SP-PCTW in weak coupling.

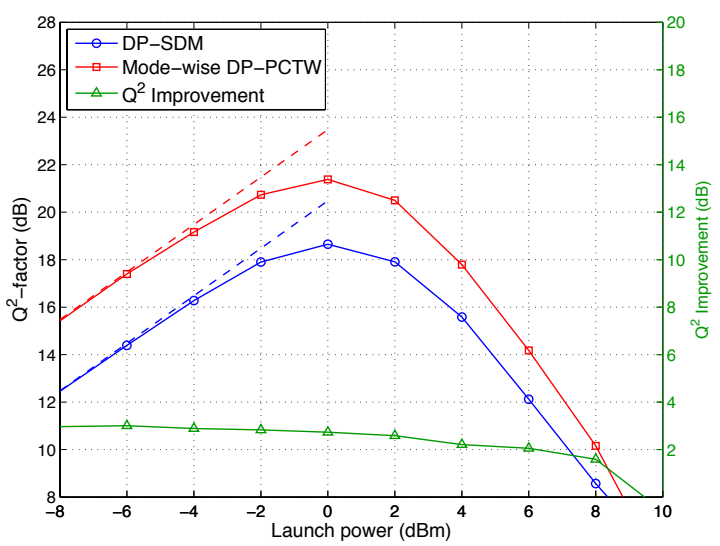

Figure 6. Mode-wise DP-PCTW in weak coupling.

Figure 7 shows an overview of all the obtained results. Compared to the original PCTW implementation, a performance improvement of $\sim 10 \mathrm{~dB}$ is achieved for PDM-wise PCTW and mode-wise SP-PCTW, and $\sim 8 \mathrm{~dB}$ for mode-wise DP-PCTW, all in the strong coupling regime. Also, the optimal launch power is increased by 5-6 $\mathrm{dB}$ in these scenarios. Regarding the weak coupling regime, performance improvement for PDM-wise PCTW is $\sim 4 \mathrm{~dB}$, mode-wise SP-PCTW shows no improvement compared to the original PCTW, and lastly, mode-wise DP-PCTW shows a performance degradation of $\sim 3 \mathrm{~dB}$.

The nonlinear distortions in a pair of PCTWs are anti correlated, therefore nonlinear distortion cancellation occurs after coherent superposition of the received signals. When resorting to an orthogonal dimension rather than polarization, the same principle applies [7]. The strong coupling regime shows better performance since 
strong interaction between modes leads to nonlinearity cancelation. The weak coupling regime shows inferior performance as expected. The performance obtained with mode-wise SP-PCTW and mode-wise DP-PCTW is poorer because PCTW is done resorting to different modes and since coupling between modes is weak, the benefit from this technique is diminished, thus the higher performance of the first scenario is due to PDM-wise twinning.

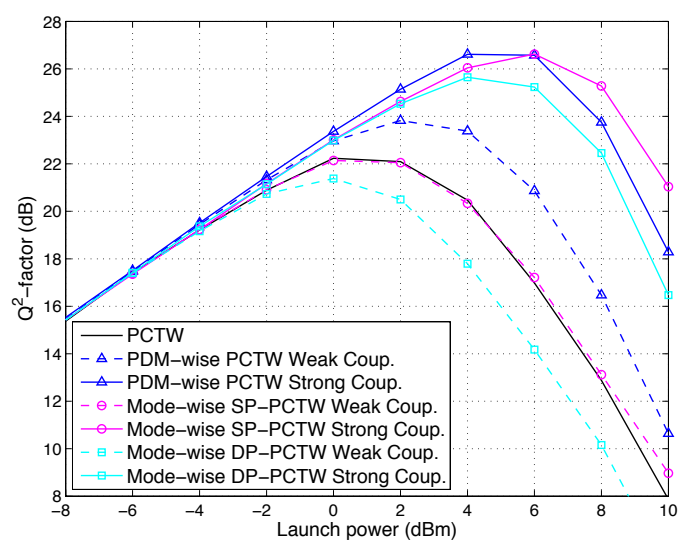

Figure 7. Calculated $Q^{2}$-factor versus launch power for single mode PCTW and all proposed multimode PCTW scenarios

\section{CONCLUSIONS}

The performance of SDM systems in different PCTW scenarios and coupling regimes has been assessed numerically. The proposed multimode PCTW configurations show a performance improvement of up to $10 \mathrm{~dB}$ in the strong coupling regime and up to $4 \mathrm{~dB}$ in the weak coupling regime, compared to the single mode PCTW case. Best performance is achieved in the strong coupling regime in all scenarios. For the weak coupling regime, PDM-wise PCTW shows the best performance, whereas mode-wise SP-PCTW shows no performance improvement, and mode-wise DP-PCTW reveals performance degradation. Also, optimal launch power is increased in most cases. These results demonstrate the effectiveness of the PCTW within SDM systems, namely for FMF based propagation.

\section{ACKNOWLEDGEMENTS}

This work was supported by FCT under the project TWAVE (EXPL/EEI-TEL/1748/2013) and in the framework of programme POCTI/FEDER with grant REEQ/1272/EEI/2005. This work was carried out with the support of the TEC4SEA research infrastructure (www.tec4sea.com).

\section{REFERENCES}

[1] Essiambre, R. and A. Mecozzi. Capacity limits in single mode fiber and scaling for spatial multiplexing. in Optical Fiber Communication Conference. 2012. Optical Society of America.

[2] Winzer, P.J. Challenges and evolution of optical transport networks. in 36th European Conference on Optical Communication (ECOC 2010), Torino, Italy, Sept. 2010.

[3] Winzer, P.J., Energy-efficient optical transport capacity scaling through spatial multiplexing. Photonics Technology Letters, IEEE, 2011. 23(13): p. 851-853.

[4] Winzer, P.J. and G.J. Foschini, MIMO capacities and outage probabilities in spatially multiplexed optical transport systems. Optics express, 2011. 19(17): p. 16680-16696.

[5] Richardson, D., J. Fini, and L. Nelson, Space-division multiplexing in optical fibres. Nature Photonics, 2013. 7(5): p. 354-362.

[6] Liu, X., et al., Phase-conjugated twin waves for communication beyond the Kerr nonlinearity limit. Nature Photonics, 2013. 7(7): p. 560-568.

[7] Liu, X., et al., Fiber-nonlinearity-tolerant superchannel transmission via nonlinear noise squeezing and generalized phase-conjugated twin waves. Journal of Lightwave Technology, 2014. 32(4): p. 766-775.

[8] Mumtaz, S., R. Essiambre, and G.P. Agrawal, Nonlinear propagation in multimode and multicore fibers: Generalization of the Manakov equations. Lightwave Technology, Journal of, 2013. 31(3): p. 398-406. 